\title{
Rigorous 3-D vectorial complex ray model applied to light scattering by an arbitrary spheroid
}

\author{
Bingqiang Sun ${ }^{1}$, George W. Kattawar ${ }^{2}$, Ping Yang ${ }^{3,1}$, Kuan Fang Ren ${ }^{4}$ \\ ${ }^{I}$ Department of Physics \& Astronomy, Texas A\&M University, College Station, TX \\ 77843 USA \\ ${ }^{2}$ Department of Physics \& Astronomy and Institute for Quantum Science and \\ Engineering, Texas A\&M University, College Station, TX 77843 USA \\ ${ }^{3}$ Department of Atmospheric Sciences, Texas A\&M University, College Station, TX \\ 77843 USA \\ ${ }^{4}$ CORIA-UMR6614, Normandie Université, CNRS, Université et INSA de Rouen Av \\ de l'Université, 76800 Saint Etienne du Rouvray, France
}

For publication in the Journal of Quantitative Spectroscopy \& Radiative Transfer

Corresponding author address: Dr. Bingqiang Sun, Department of Physics \& Astronomy, Texas A\&M University, College Station, TX 77843, USA. Email: bqsun@physics.tamu.edu 


\begin{abstract}
After a ray bundle passes a curved surface, the equal-phase wavefront associated with the refracted rays will be distorted. Consequently, the cross-section of a ray bundle with a curved wavefront during propagation in a homogeneous medium will vary with the raybundle propagation distance. Moreover, the phase of a ray bundle with convergent wavefront will undergo a phase shift of $\pi / 2$ with each passage of a focal line. The contribution to the scattering amplitude by a ray bundle after passing a scatterer is determined by three elements: the cross-section variation of its wavefront, the total phase, and the refraction coefficients determined by Fresnel equations. In the geometric optics regime, the aforesaid three elements caused by a curved surface can be systematically quantified in terms of the vectorial complex ray-tracing technique. In this study, rigorous vectorial complex ray-tracing calculations are conducted for light scattering by a general spheroid and the results are validated in comparison with the benchmarks provided by the rigorous T-matrix method.
\end{abstract}

Keywords: light scattering, vectorial complex ray-tracing, spheroid. 


\section{Introduction}

The single-scattering properties of homogeneous spheres or multiple spherical shells for given sizes can be analytically obtained by the Lorenz-Mie theory [1, 2]. Light scattering by small-sized arbitrary scatterers can be modeled by numerically accurate methods including the discrete dipole approximation method (DDA) [3-5], finite difference time domain method (FDTD) [6-8], and pseudo-spectral time domain method (PSTD) [9-11]. For small-to-moderate scatterers with rotational symmetry, two effective realizations of the T-matrix method, namely, the extended boundary condition method (EBCM) [12-14] and invariant-imbedding T-matrix method (IITM) $[15,16]$, can facilitate the singlescattering property computations much more accurately and effectively than numerical methods, particularly in the case of randomly oriented particles. For a scatterer with its characteristic dimension much larger than the incident wavelength, the geometric optics method can provide a reasonably accurate approximation [17-19]. In some geometric optics methods, the physical-optics effect, such as accurately mapping the near-field to far-field (e.g. [18]), surface waves (e.g. [20]), tunneling rays (e.g. [21]), and, caustics (e.g. [22]), has been taken into consideration.

For a scatterer with a curved surface, the wavefront of a refracted wave is distorted. Compared to the conventional geometric optics method, a divergence factor must be introduced to represent the effect caused by wavefront distortion [1, 20, 23-25]. Consequently, the variations in the phase and cross-section of a ray bundle will give rise to additional complexity in comparison with ray tracing in the case of a scatterer with facet faces $[1,26]$. The effect caused by wavefront distortion has been theoretically and experimentally studied using the vectorial complex ray model in 2D propagation where 
the incident and refracted rays remain in the same plane [25, 27-29]. In this study, the vectorial complex ray-tracing technique [22] including the wavefront distortion effect is implemented for the 3D case for a general spheroid; however, the present study is still restricted to the pure geometric optics method.

The present study focuses on the scattering phase matrix, which includes all the scattering information and can be obtained from the amplitude scattering matrix $[1,2$, 14]. The amplitude scattering matrix $\mathbf{S}$ defined with respect to the scattering plane spanned by the incident and scattered directions shown in Fig. 1 is given in the form [1, 2]:

$$
\left(\begin{array}{l}
E_{\|}^{\mathrm{sca}} \\
E_{\perp}^{\mathrm{sca}}
\end{array}\right)=\frac{\exp (i k r)}{-i k r}\left(\begin{array}{ll}
S_{11} & S_{12} \\
S_{21} & S_{22}
\end{array}\right)\left(\begin{array}{l}
E_{\|}^{\mathrm{inc}} \\
E_{\perp}^{\mathrm{inc}}
\end{array}\right)
$$

where $\mathrm{r}$ is the distance from the origin to the point of observation; The quantities $E_{\|}$and $E_{\perp}$ are the parallel and perpendicular components of the electric field, decomposed with respect to the scattering plane. The superscripts "inc" and "sca" denote the incident and scattered quantities.

\section{Method}

\subsection{Scattering amplitude}

Consider a scatterer of refractive index $m$ illuminated by a plane wave with wavelength $\lambda$, and assume a ray bundle with a plane wavefront to impinge on the surface of the scatterer with a solid angle element $d \Omega_{\text {int }}$, which is defined in terms of the area of the image of a Gauss map of the corresponding incident area. Consequently, a scattered ray bundle will spread into a solid angle $d \Omega_{\text {sca }}$, which is defined as the area of the image of a 
Gauss map of the corresponding wavefront [30]. The intensity of the scattered light can be formulated as [1]:

$$
I_{S}=T_{f} I_{0} \frac{\cos \theta_{i, 1} d \Omega_{\mathrm{int}} / K_{\mathrm{int}}}{d \Omega_{\mathrm{sca}} / K_{\mathrm{sca}}}
$$

where $I_{0}$ and $I_{S}$ are respectively the incident and scattered intensities; $K_{\text {int }}$ and $K_{\text {sca }}$ are the Gaussian curvatures of the surface of the scatterer at the incident point and the wavefront of the scattered light, respectively; $\theta_{i, 1}$ is the initial incident angle. The transmission coefficients $T_{f}$ are related to the Fresnel equations and incident and refracted angles by

$$
T_{f, p}=\left\{\begin{array}{l}
\left|r_{1}\right|^{2}, \quad p=0 \\
\left(\frac{\cos \theta_{t, p+1}}{\cos \theta_{i, p+1}}\left|t_{p+1}\right|^{2}\right)\left(\left.|| \prod_{n=2}^{p} r_{n}\right|^{2} \mid\left(\frac{\cos \theta_{t, 1}}{\cos \theta_{i, 1}}\left|t_{1}\right|^{2}\right), \quad p \geq 1\right.
\end{array}\right.
$$

where the subscript $p$ is the order of the emergent ray (Fig. 2), $r_{n}$ and $t_{n}$ are respectively the Fresnel reflection and refraction coefficients at the $n^{\text {th }}$ interaction of the ray with the particle surface. $\theta_{i, n}$ and $\theta_{t, n}$ are the incident angle and the refracted angle of the $n^{\text {th }}$ interaction of the ray with the particle surface. In the far field, the scattering wave is spherical, $K_{\text {sca }}=1 / r^{2}$, and the scattering matrix for a ray bundle can be formulated in the form:

$$
\mathbf{S}=\sum_{p=0}^{\infty} \exp \left(i \zeta_{p}\right) \sqrt{D_{p}} \mathbf{U}_{\mathrm{p}} \mathbf{G}
$$

where $\zeta_{p}$ is the phase of the scattered wave, which consists of the phase shift due to the length of optical path relative to the reference ray, the dot-dashed line in Fig. 2, and the 
phase shift due to the focal points/lines [1]; $\mathbf{U}_{\mathrm{p}}$ is a coefficient matrix, which is related to the Fresnel coefficients and the corresponding rotation matrices. $\mathbf{G}$ is a rotation matrix related to the incident frame of reference and the scattering plane defined by[31]:

$$
\boldsymbol{G}=\left(\begin{array}{cc}
\hat{e}_{Y} \cdot \hat{e}_{\|}^{i n t} & \hat{e}_{Y} \cdot \hat{e}_{\perp}^{i n t} \\
\hat{e}_{X} \cdot \hat{e}_{\|}^{i n t} & \hat{e}_{X} \cdot \hat{e}_{\perp}^{i n t}
\end{array}\right)
$$

where the $\hat{e}_{X}$ and $\hat{e}_{Y}$ represent the unit vectors along $\mathrm{X}$-axis and $\mathrm{Y}$-axis directions in the incident frame of reference and, $\hat{e}_{\|}^{\text {int }}$ and $\hat{e}_{\perp}^{\text {int }}$ the unit vectors parallel and perpendicular to the scattering plane, as shown in Fig. 1 . The divergence factor is defined by [1, 25]:

$$
D_{p}=\frac{\cos \theta_{t, p+1} \cos \theta_{t, 1}}{\cos \theta_{i, p+1}} \frac{d \Omega_{i n t} / K_{i n t}}{d \Omega_{s c a}}=\frac{1}{K_{t, 1}} \prod_{n=2}^{p+1} \frac{K_{i, n}}{K_{t, n}}
$$

where $K_{i, n}$ and $K_{t, n}$ are respectively the corresponding Gaussian curvature of the wavefront of the incident and refracted rays at the $n^{\text {th }}$ interaction of the ray with the particle surface. The total scattering matrix associated with an incident ray can be formally expressed as:

$$
\mathbf{S}=\mathbf{S}_{d i f f}+\sum_{j} \mathbf{S}_{j}
$$

where $\mathbf{S}_{\text {diff }}$ is the scattering matrix of diffraction, which can be obtained in terms of the surface-mapping technique $[18,32]$; the summation is to cover all the rays illuminating the scatterer along the incident direction; and $\mathrm{j}$ is used to count the number of ray bundles.

\subsection{Scattering solid angle}

We define a differential direction vector as: 


$$
\vec{\vartheta}_{d}=d \theta \hat{e}_{\theta}-\sin \theta d \phi \hat{e}_{\phi}
$$

where $\theta$ and $\phi$ are the zenith and azimuthal angles relative to the incident frame of reference, as shown in Fig. 1, and, $d \theta$ and $d \phi$ are the corresponding differential angles and the subscript $\mathrm{d}$ denotes a differential quantity. For a ray bundle, $m k_{0} \vec{\vartheta}_{d}\left(k_{0}\right.$ the wavenumber in the surrounding medium) is actually the projection of the wave vector $\vec{k}$ of a paraxial ray onto a wave plane $P$ perpendicular to its axial ray, as shown in Fig. 3 . The position of the paraxial ray relative to the incident point $O$ can be defined by the vector $\vec{\sigma}_{d}$ in the tangent plane of the wave surface $P$, and the vector $\vec{\rho}_{d}$ on the tangent plane of the scatterer surface $\sum$, as shown in Fig. 4 . The vectors $\vec{\sigma}_{d}$ and vector $\vec{\rho}_{d}$ are related in terms of a projection matrix $\mathbf{B}$ :

$$
\vec{\sigma}_{d}=\mathbf{B} \vec{\rho}_{d}
$$

The relationship between the direction vector $\vec{\vartheta}_{d}$ and the position vector $\vec{\sigma}_{d}$ can be bridged by the symmetric curvature matrix $\mathbf{Q}$ of the wavefront of a ray bundle [26]:

$$
\vec{\vartheta}_{d}=\mathbf{Q} \vec{\sigma}_{d}
$$

The ray bundle, the dashed arrows in Figs. 3 and 4, can be described by a matrix composed of two vectors defined by $\mathbf{L}=\left(\begin{array}{c}\vec{\sigma} \\ m \vec{\vartheta}\end{array}\right)[26]$.

When a ray bundle proceeds in a homogeneous medium from the point $n$ - 1 to point $n$, as shown in Fig. 3, the direction vector $\vec{\vartheta}_{d}$ remains the same while the displacement vector $\vec{\sigma}_{d}$ increase as $d_{n, n-1} \vec{\vartheta}_{d}$, where $d_{n, n-1}$ is the distance from the point $n-1$ to point 
$n$. Correspondingly, the transformation of the ray matrix between the two successive points in a homogeneous medium can be given as [26]:

$$
\mathbf{L}_{n}=\mathbf{M}_{n, n-1} \mathbf{L}_{n-1}
$$

where the transformation matrix $\mathbf{M}_{n, n-1}$ is defined by

$$
\mathbf{M}_{n, n-1}=\left(\begin{array}{cc}
1 & d_{n, n-1} / m \\
0 & 1
\end{array}\right)
$$

When a ray bundle passes a refracting surface, the boundary conditions of Maxwell's equations require the phase of the refracted ray to be the same as that of the incident ray. Moreover, the position vector $\vec{\rho}_{d}$ must be the same before and after the refraction. Consequently, the transformation of the refracted/reflection ray matrix $\mathbf{L}_{n, \text { ref }}$ to the refracted ray matrix $\mathbf{L}_{n, \text { int }}$ is given by

$$
\mathbf{L}_{n, \text { ref }}=\mathbf{M}_{n} \mathbf{L}_{n, \text { int }}
$$

where the matrix $\mathbf{M}_{n}$ is given by

$$
\mathbf{M}_{n}=\left(\begin{array}{cc}
\mathbf{B}_{t, n} \mathbf{B}_{i, n}^{-1} & 0 \\
\left(m_{t, n} \cos \theta_{t, n}-m_{i, n} \cos \theta_{i, n}\right) \mathbf{B}_{t, n}^{-T} \mathbf{C}_{n} \mathbf{B}_{i, n}^{-1} & \mathbf{B}_{t, n}^{-T} \mathbf{B}_{i, n}^{T}
\end{array}\right)
$$

where $\mathbf{C}_{n}$ is the symmetric curvature matrix of the scatterer surface $\sum$ at the incident point $\mathrm{O}$; the subscript $\mathrm{t}$ and $\mathrm{i}$ still denote the refraction and incident quantities (the reflection can be treated as a special case of the refraction). A linear relation exists between the $\mathrm{p}$-th order scattered ray and the incident ray bundle vectors in the narrow beam approximation [26]:

$$
\mathbf{L}_{\mathrm{sca}, p}=\mathbf{M L}_{\mathrm{int}}=\left[\prod_{n=2}^{p+1}\left(\mathbf{M}_{n} \mathbf{M}_{n, n-1}\right)\right] \mathbf{M}_{1} \mathbf{L}_{\mathrm{int}}
$$


The divergence factor and, the principal curvatures and the eigenvectors of the refracted wavefront of a ray bundle can be obtained in terms of Eqs. (10) and (13) $[25,26]$.

\subsection{Plane wave incident}

If the incident wave is a plane wave, $\vec{\vartheta}_{d \text {,int }}$ will be zero. In terms of Eq. (14), the scattering solid angle can be expressed as:

$$
m_{0} \vec{\vartheta}_{d, \text { sca }}=\mathbf{M}_{21} \vec{\sigma}_{d, \text { int }}
$$

where $m_{0}$ is the refractive index of the surrounding medium and $\mathbf{M}_{21}$, a 2 by 2 matrix, is the elements of matrix $\mathbf{M}$ in Eq. (14), that is,

$$
M=\left(\begin{array}{ll}
M_{11} & M_{12} \\
M_{21} & M_{22}
\end{array}\right)
$$

Moreover, the vector $\vec{\rho}_{d, \Sigma}$ can have a similar expression as Eq. (10) on the scatterer surface in the incidence:

$$
\vec{\vartheta}_{d, \Sigma}=\mathbf{C}_{1} \vec{\rho}_{d, \Sigma}
$$

where $\vec{\vartheta}_{\Sigma}$ is the vector on the scatterer surface. In terms of Eqs. (9) and (17), the incident position vector $\vec{\sigma}_{d, \text { int }}$ can be expressed as:

$$
\vec{\sigma}_{d, \text { int }}=\mathbf{B}_{i, 1} \mathbf{C}_{1}^{-1} \vec{\vartheta}_{d, \Sigma}
$$

In terms of Eqs. (15) and (18), the transformation between incident and scattering solid angle element can be constructed as:

$$
\vec{\vartheta}_{d, \mathrm{sca}}=\frac{1}{m_{0}} \mathbf{M}_{21} \mathbf{B}_{i, 1} \mathbf{C}_{1}^{-1} \vec{\vartheta}_{d, \Sigma}
$$


In terms of Eqs. (19) and (7), the vector $\vec{\vartheta}$ and the scattering matrix $\mathbf{S}$ can be formalized in a differential form and $\mathbf{S}=\mathbf{S}\left(\vec{\vartheta}_{\mathrm{sca}}\right)[30,33]$

Once the resolution of the incident ray bundle $\left(\Delta \theta_{\text {int }}, \sin \theta_{\text {int }} \Delta \phi_{\text {int }}\right)$ is given, the resolution of the corresponding scattering ray bundles $\left(\Delta \theta_{s c a}, \sin \theta_{s c a} \Delta \phi_{s c a}\right)$ can be achieved in terms of Eq. (19). The scattering range at each scattering order $\mathrm{p}(\mathrm{p}=0,1,2, \ldots)$ can be obtained in terms of the scattering angles of the central ray $\left(\theta_{s c a}, \phi_{s c a}\right)$ and the resolution of the ray bundles, that is, $\left(\theta_{s c a} \pm \Delta \theta_{s c a} / 2, \phi_{s c a} \pm \Delta \phi_{s c a} / 2\right)$. Inside this scattering range, the phase $\zeta_{p}$, the divergence factor $D_{p}$, and the coefficient matrix $\mathbf{U}_{p}$ can be obtained by the accumulating results from the order 0 to the order $\mathrm{p}$. The scattering matrix is straightforward in terms of all these quantities inside the scattering range. When the incident resolutions are covering all the illuminated sides of the scatterers, the summation in Eq. (7) is eventually accomplished. For any scattering angle, the diffracted scattering matrix $\mathbf{S}_{\text {diff }}$ of spheroids can be analytically obtained [32]. Correspondently, the total scattering matrix can be given in terms of Eq. (7). Note that the present study is still confined to the pure geometric optics; thus the outgoing direction of a ray bundle is exactly the direction in the far field, that is, no ray spreading is considered. In the simulation, an empirical value, 15 , is taken for the internal reflections of each ray bundle.

\section{Simulation results}

The II-TM $[15,16]$ is a volume integral method to get the T-matrix of a scatterer in terms of the invariant imbedding technique. It is employed to validate the computational results of the geometric optics method for scatterers with distorted wavefronts (denoted as 
DGOM in figure legends). The convergence of the II-TM is empirically determined, so the many-body iterative T-matrix (MBIT) method [34, 35], obtained by dividing a scatterer into several sub-scatterers and obtaining the results in terms of an iteration technique, is used to validate II-TM with a large characteristic length. In the case of random orientations, the results calculated by the improved geometric optics method (IGOM) [18], taking physical-optics effects into consideration in the near-field to farfield mapping, is also illustrated to show the agreement. In the following figures, Mueller matrix elements are shown only as the function of the zenith scattering angles from $0^{0}$ to $180^{\circ}$ since the azimuthal dependence has been averaged from $0^{\circ}$ to $360^{\circ}$.

Figure 5 shows the comparisons of the scattering matrix elements of a circular cylinder calculated by the II-TM and MBIT. The incident wavelength is $0.628 \mu \mathrm{m}$. The height and the diameter of the bottom surface of the cylinder are $60 \mu \mathrm{m}$ and $4 \mu \mathrm{m}$, respectively. The angle between the incident direction and the cylinder symmetry axis is $30^{\circ}$, as illustrated in Fig. 5. The refractive index of the cylinder is $1.33+\mathrm{i} 0.0$. The computational feasibility of the II-TM is closely related to the radius of the circumscribed sphere of a scatterer. The maximum dimension of the cylinder is almost 100 times of the incident wavelength. The two methods agree with each other even for a large size.

Figure 6 shows the comparisons of the scattering matrix elements of a sphere calculated by the Lorenz-Mie method (denoted as MIE) and DGOM. The incident wavelength is $0.628 \mu \mathrm{m}$. The radius of the sphere is $60 \mu \mathrm{m}$ and the refractive index is 1.33+i0.0. For $\mathrm{P}_{11}$ the DGOM results are shifted by a factor of 100 while, for other elements (e.g., $\mathrm{P}_{12} / \mathrm{P}_{11}$ ), they are shifted by 2 for clarity. For homogeneous spheres, the ray tracing process always remains in the same plane. At caustic points, where the 
divergence factor will be infinity, the geometric optics method, including DGOM, is not correct. There are two steep jumps close to the primary and secondary rainbow angles (caustics) in $\mathrm{P}_{11}$; however, others angles agree well. For other scattering matrix elements, the DGOM results follow the same trend as the MIE results although there are some discrepancies in the oscillations.

Figure 7 shows the comparisons of the scattering matrix elements of a prolate spheroid in a fixed orientation calculated by the II-TM and DGOM. The incident wavelength is $0.628 \mu \mathrm{m}$. The lengths of its semi-major and semi-minor axes are $30 \mu \mathrm{m}$ and $6 \mu \mathrm{m}$. The angle between the incident direction and the spheroid symmetry axis is $60^{\circ}$. The refractive index of the spheroid is $1.33+\mathrm{i} 0.0$. Close to the caustic points, the geometric optics method will not work. Correspondently, there are several discrepancies for $\mathrm{P}_{11}$ between $90^{\circ}$ and $120^{\circ}$. The backscattering has some errors for elements $\mathrm{P}_{22} / \mathrm{P}_{11}$, $\mathrm{P}_{33} / \mathrm{P}_{11}$, and $\mathrm{P}_{44} / \mathrm{P}_{11}$.

Figure 8 shows the comparisons of the scattering matrix elements of an oblate spheroid in a fixed orientation calculated by the II-TM and DGOM. The lengths of its semi-major and semi-minor axes are $30 \mu \mathrm{m}$ and $6 \mu \mathrm{m}$. The incident angle is $45^{\circ}$. All other parameters are the same as Fig. 7. At caustic points, the DGOM for $\mathrm{P}_{11}$ has some discrepancies, compared with II-TM. The agreement of other elements is better than the counterparts of Figure 7.

Figure 9 shows the comparisons of the scattering matrix elements of a prolate spheroid calculated in random orientation by the II-TM, IGOM, and DGOM. The incident wavelength is $0.628 \mu \mathrm{m}$. The lengths of its semi-major and semi-minor axes are $20 \mu \mathrm{m}$ and $6 \mu \mathrm{m}$. The refractive index of the spheroid is $1.33+\mathrm{i} 0.0$. Under random 
orientation condition, the interference of outgoing rays can be neglected and the effects caused by the wavefront distortion can be also considered negligible. The DGOM and IGOM have good agreement even though IGOM has taken physical optics into consideration. Compared to the II-TM, both the IGOM and DGOM results show some discrepancies in the backscattering directions.

Figure 10 shows the comparisons of the scattering matrix elements of an oblate spheroid calculated in random orientation by the II-TM, IGOM, and DGOM. The incident wavelength is $0.628 \mu \mathrm{m}$. The lengths of its semi-major and semi-minor axes are $44.46 \mu \mathrm{m}$ and $39 \mu \mathrm{m}$. The refractive index of the spheroid is $1.33+\mathrm{i} 0.0$. Compared to IITM, the agreement of the IGOM and DGOM is getting better with a larger size than the one used in Fig. 9.

Figure 11 shows the comparisons of the scattering matrix elements of a prolate spheroid calculated in random orientation by the II-TM, IGOM, and DGOM. The incident wavelength is $0.628 \mu \mathrm{m}$. The lengths of its semi-major and semi-minor axes are $10 \mu \mathrm{m}$ and $4 \mu \mathrm{m}$. The refractive index of the spheroid is $1.53+\mathrm{i} 0.008$. The agreement between the approximate methods, DGOM and IGOM, and the accurate method, the IITM is good when the scatterer has a strong absorption. All other elements except $\mathrm{P}_{11}$ show similar Rayleigh-Gans scattering features when strong absorption is involved in conjunction with a large size. In this situation, the diffraction and external reflection components will dominate the scattering patterns. Moreover, the scattering feature generated by external reflection on a large convex scatterer in random orientation is the same as the scattering feature generated by external reflection on a large sphere with the 
same material and surface conditions [1]. These Rayleigh-Gans scattering features are actually from external reflection of an equivalent sphere.

\section{Conclusions}

Within the framework of the conventional geometric optics method, the present study considers the wavefront distortion effect, represented by a divergence factor, after a ray bundle passes a curved surface. Moreover, the wavefront distortion in the refraction process can cause ray convergence or divergence that can be traced for every scattering order. The scattering amplitude and scattering matrix can be obtained with the geometric optics method by considering the wavefront distortion effect. The simulation results are compared to the II-TM under both fixed and random orientation conditions and IGOM under random orientation condition, and good agreement is achieved.

\section{Acknowledgements}

The authors are grateful to Dr. L. Bi for help with the invariant-imbedding T-matrix light scattering computational program used in this study. Computational support was provided by the Texas A\&M Supercomputing Facility. Bingqiang Sun was supported by ONR Coastal Geophysics. George Kattawar acknowledges support by the National Science Foundation Grant \# OCE-1130906. Ping Yang acknowledges the support by a NSF grant (AGS-1338440) and partially by the endowment funds (TAMU512231-10000) related to the David Bullock Harris Chair in Geosciences at the College of Geosciences, Texas A\&M University. The authors thank the anonymous reviewers for constructive comments. 


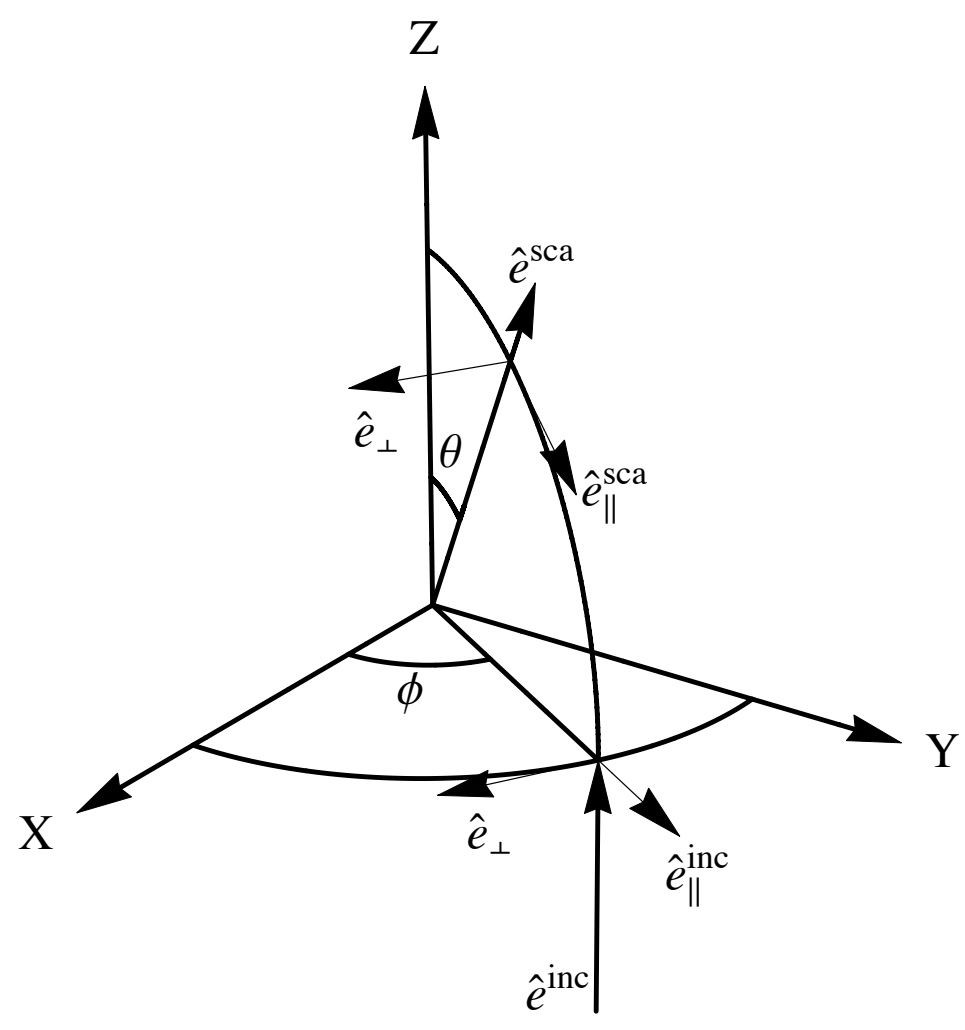

Figure 1. Scattering plane spanned by the incident and scattered directions, i.e., $\hat{e}^{\text {inc }}$ and $\hat{e}^{s c a}$, in the incident frame of reference, where the incident direction is along the $\mathrm{z}$-axis. $\theta$ and $\phi$ are the zenith and azimuthal angles in the incident frame of reference. The subscripts $\|$ and $\perp$ are the corresponding parallel and perpendicular components with respect to the scattering plane, respectively. 


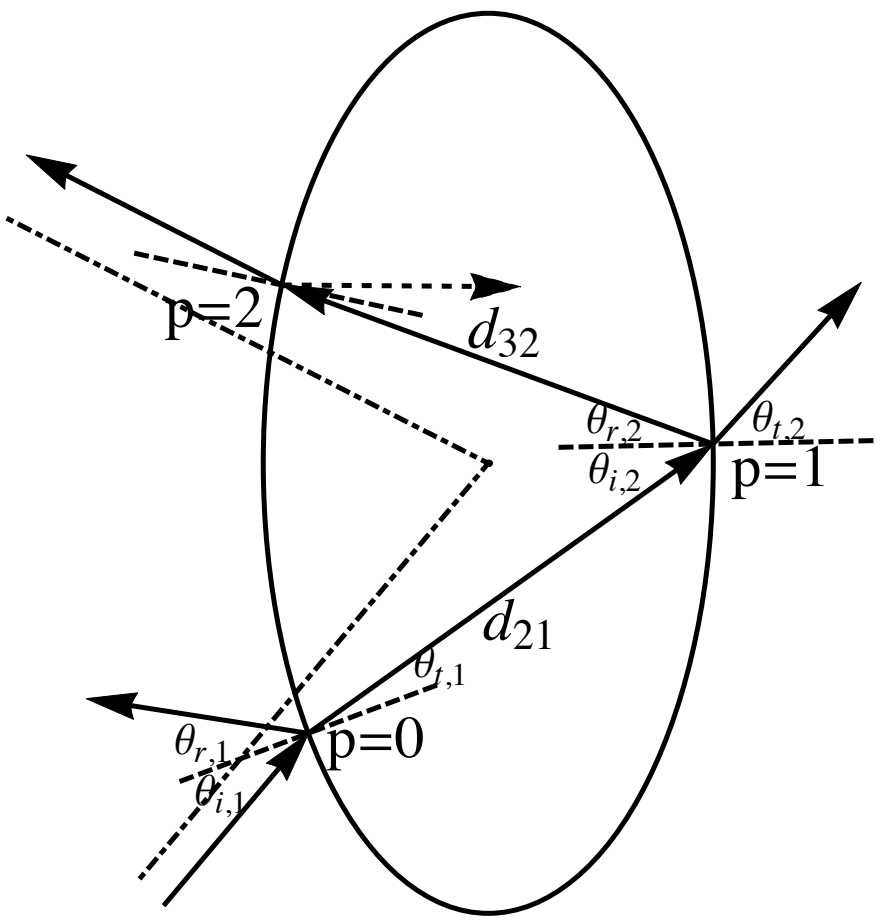

Figure 2. Schematic diagram illustrating the ray-tracing process in the case of a scatterer with curved surface. The ray tracing order $\mathrm{p}$ is defined in the axial ray of a ray bundle. $\theta_{i}$, $\theta_{r}$, and $\theta_{t}$ are incident, reflected, and refracted angles, respectively, shown for the cases of the first and second interactions ( $\mathrm{p}=0$ and 1 ) with the particle surface. Dashed lines are normal directions of different ray tracing orders and dot-dashed lines are reference lines. $d_{p, p-1}$ is propagation distance from the points ( $\left.\mathrm{p}-1\right)$ to $\mathrm{p}$. 


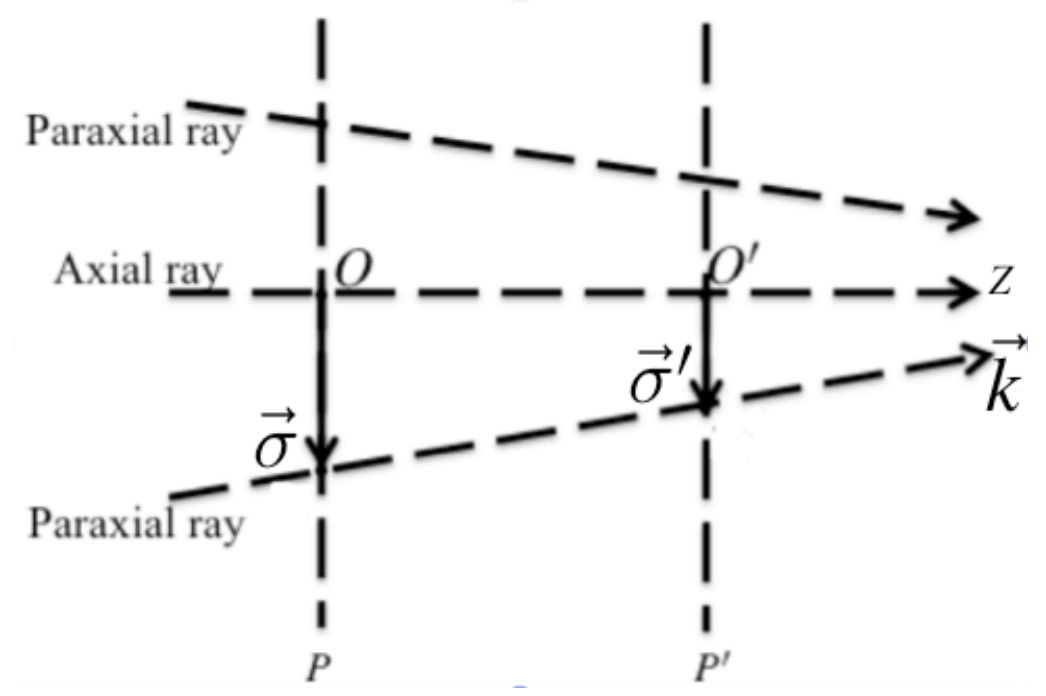

Figure 3. Schematic diagram illustrating the propagation of a ray bundle in a homogeneous medium. $O Z$ is the direction of the axial ray. $\mathrm{P}$ indicates the plane perpendicular to $O Z \cdot \vec{k}$ is the wave vector of one of the paraxial rays and $\vec{\sigma}$ the displacement vector of the paraxial ray projected on the wave plane P. Primed quantities represent the quantities at different position. 


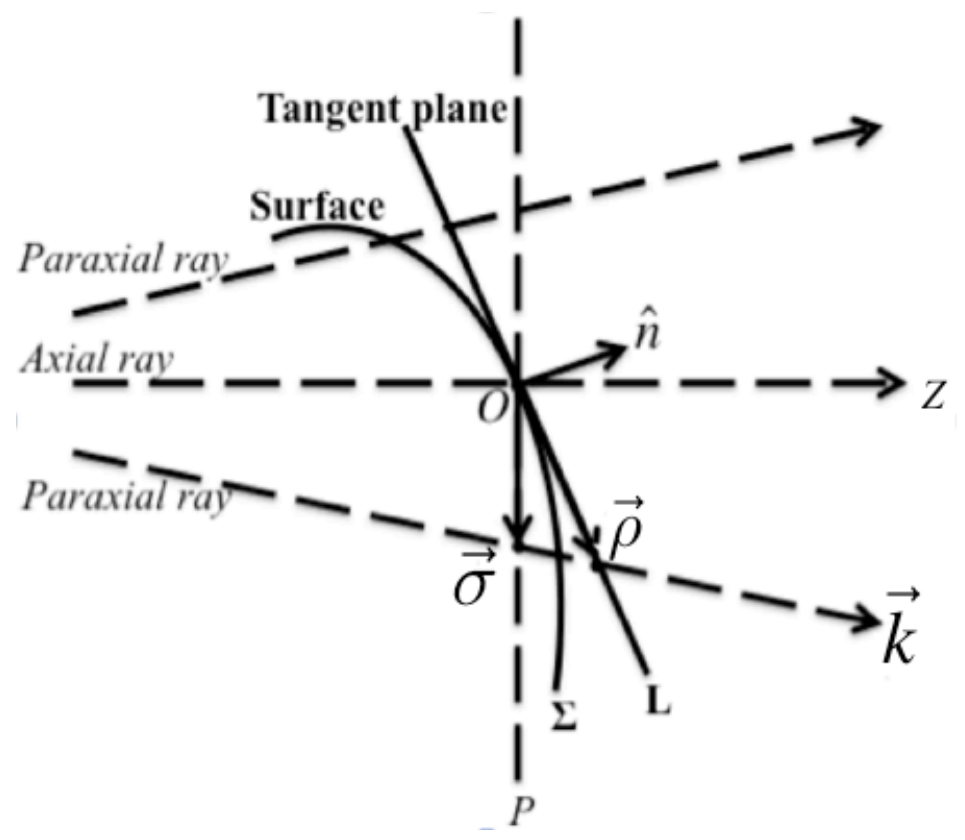

Figure 4. Schematic diagram of a ray bundle just before refraction, consisting of an axial ray and paraxial rays. $O Z$ is the direction of the axial ray, where $O$ is the intersection point with the surface $\sum$ of a scatterer. $\hat{n}$ is normal to the surface $\sum$ and $\mathrm{L}$ is its tangent plane. $\vec{\rho}$ is the displacement vector of a paraxial ray in the tangent plane L. All other quantities are the same as in Figure 3. 

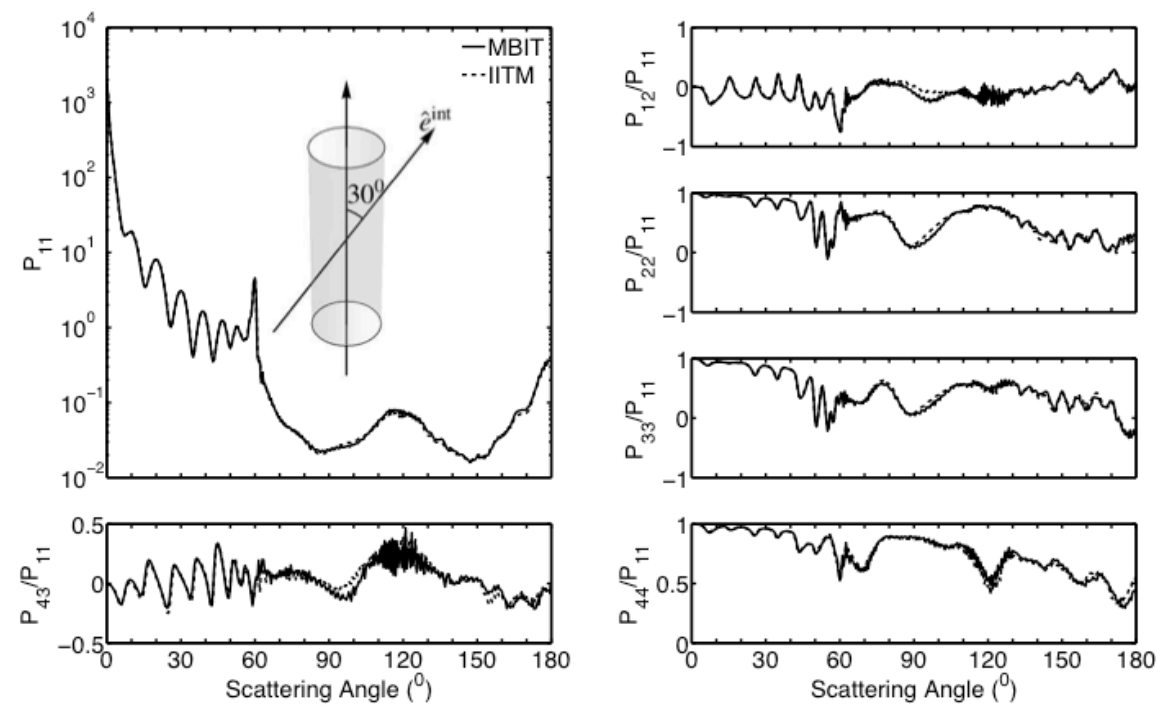

Figure 5. Comparisons of the scattering matrix elements of a circular cylinder calculated by II-TM and MBIT. The incident wavelength is $0.628 \mu \mathrm{m}$. The height and the diameter of the bottom surface of the cylinder are $60 \mu \mathrm{m}$ and $4 \mu \mathrm{m}$. The incident angle is $30^{\circ}$. The refractive index of the cylinder is $1.33+\mathrm{i} 0.0$. 

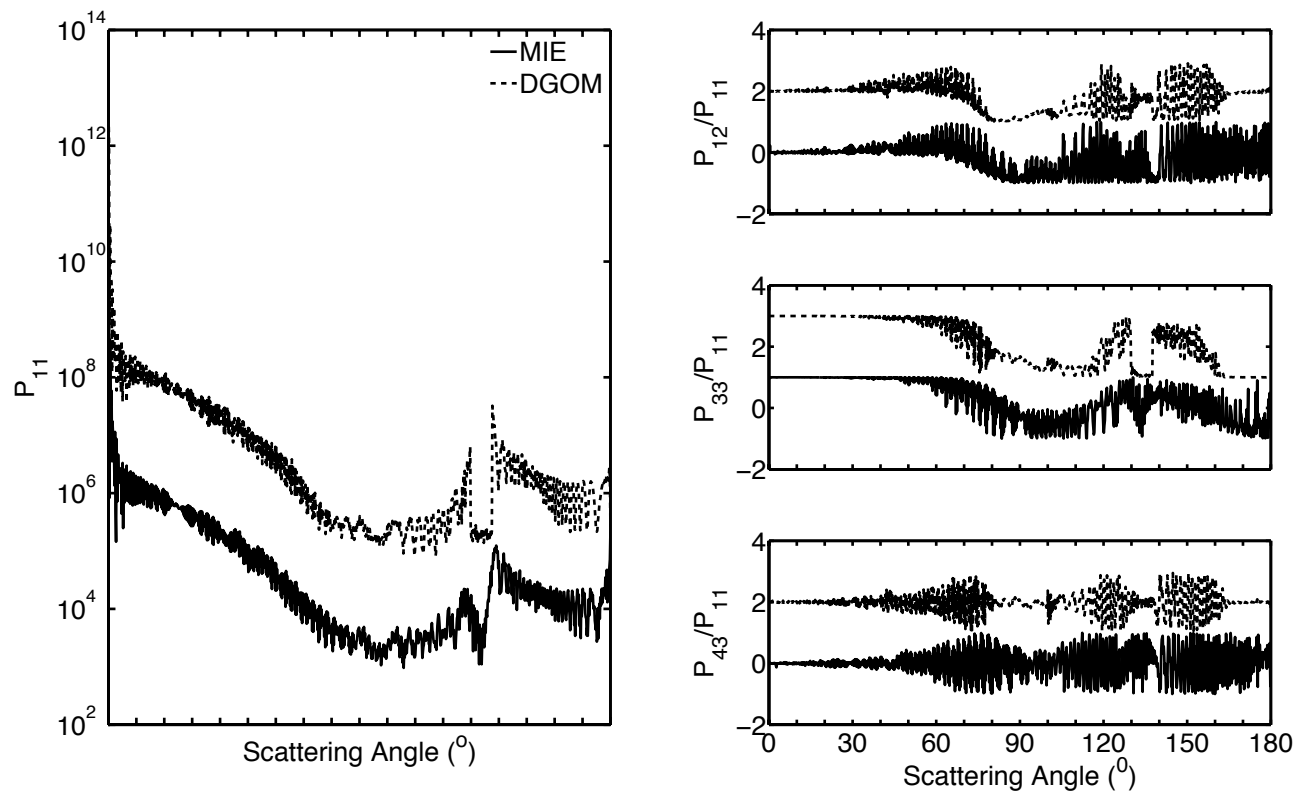

Figure 6. Comparison of the scattering matrix elements of a sphere calculated by the Lorenz-Mie theory (denoted as MIE) and the geometric optics methods for distorted wavefront (denoted as DGOM in legends, hereafter). The incident wavelength is 0.628 $\mu \mathrm{m}$. The radius of the sphere is $60 \mu \mathrm{m}$ and the refractive index is $1.33+\mathrm{i} 0.0$. For $\mathrm{P}_{11}$ the DGOM results are multiplied 100 for clarity while for other elements, they are added 2 for clarity. 

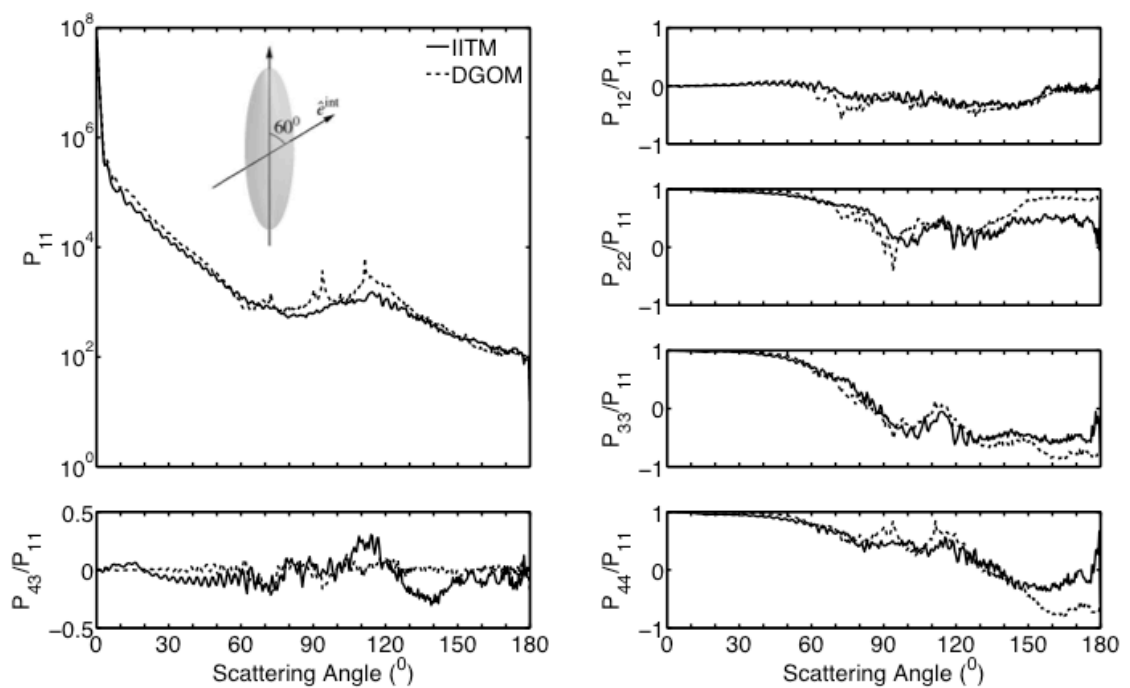

Figure 7. Comparisons of the scattering matrix elements of a prolate spheroid in a fixed orientation calculated by II-TM and DGOM. The incident wavelength is $0.628 \mu \mathrm{m}$. The lengths of its semi-major and semi-minor axes are $30 \mu \mathrm{m}$ and $6 \mu \mathrm{m}$. The incident angle is $60^{\circ}$. The refractive index of the spheroid is $1.33+\mathrm{i} 0.0$. 

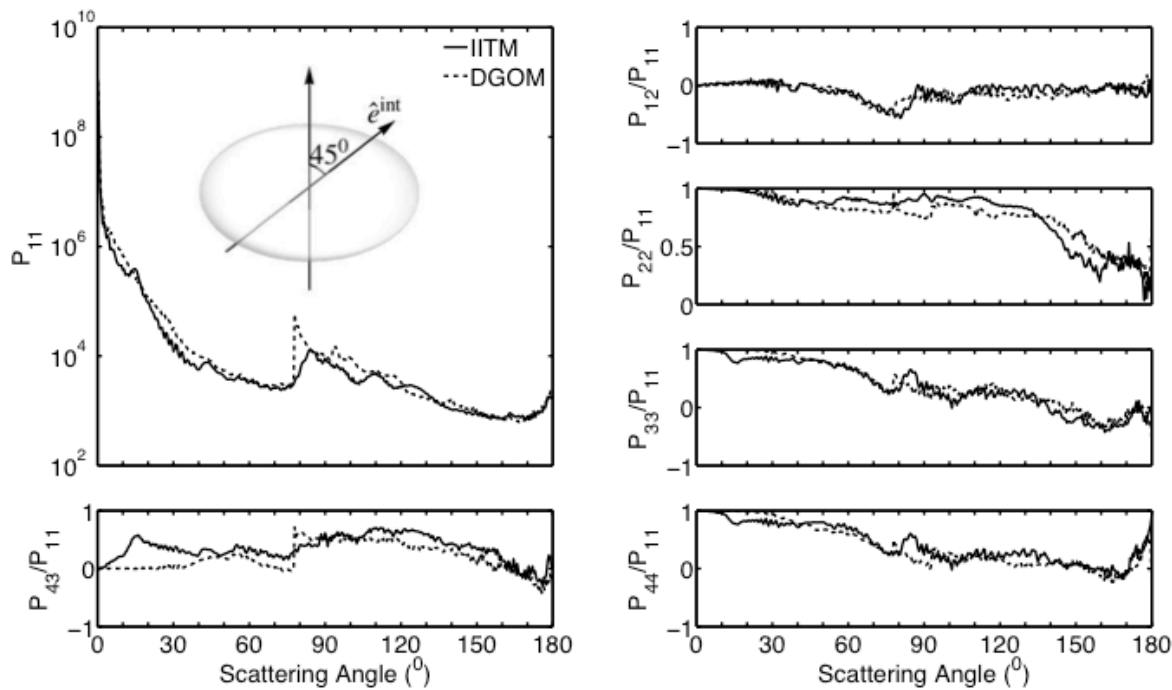

Figure 8. Comparisons of the scattering matrix elements of an oblate spheroid in a fixed orientation calculated by IITM and DGOM. The lengths of its semi-major and semiminor axes are $30 \mu \mathrm{m}$ and $6 \mu \mathrm{m}$ (the inset of P11 is not characterized by real size scales). The incident angle is $45^{\circ}$. All other parameters are the same as Fig. 7. 

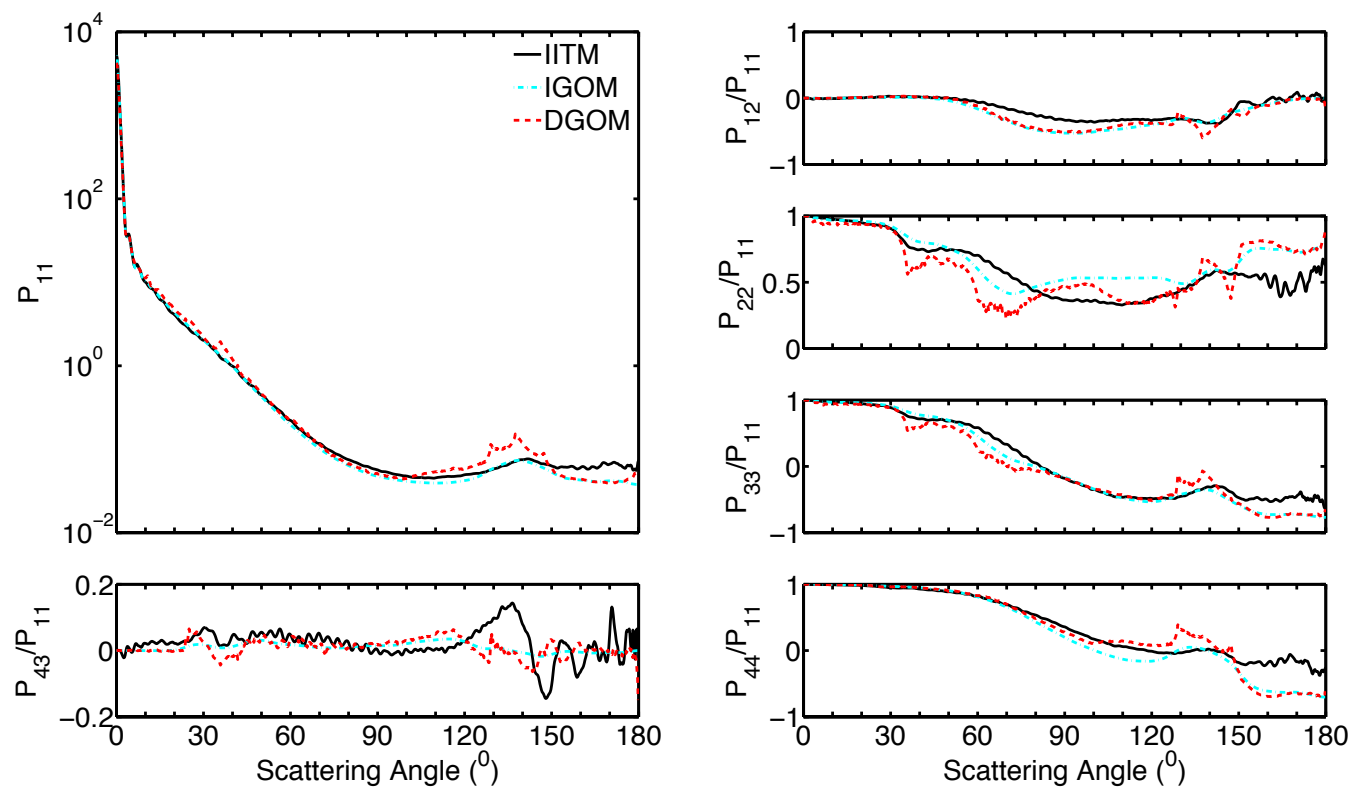

Figure 9 Comparisons of the scattering matrix elements of a prolate spheroid calculated in random orientation by IITM, IGOM, and DGOM. The incident wavelength is $0.628 \mu \mathrm{m}$. The lengths of its semi-major and semi-minor axes are $20 \mu \mathrm{m}$ and $6 \mu \mathrm{m}$. The refractive index of the spheroid is $1.33+\mathrm{i} 0.0$. 

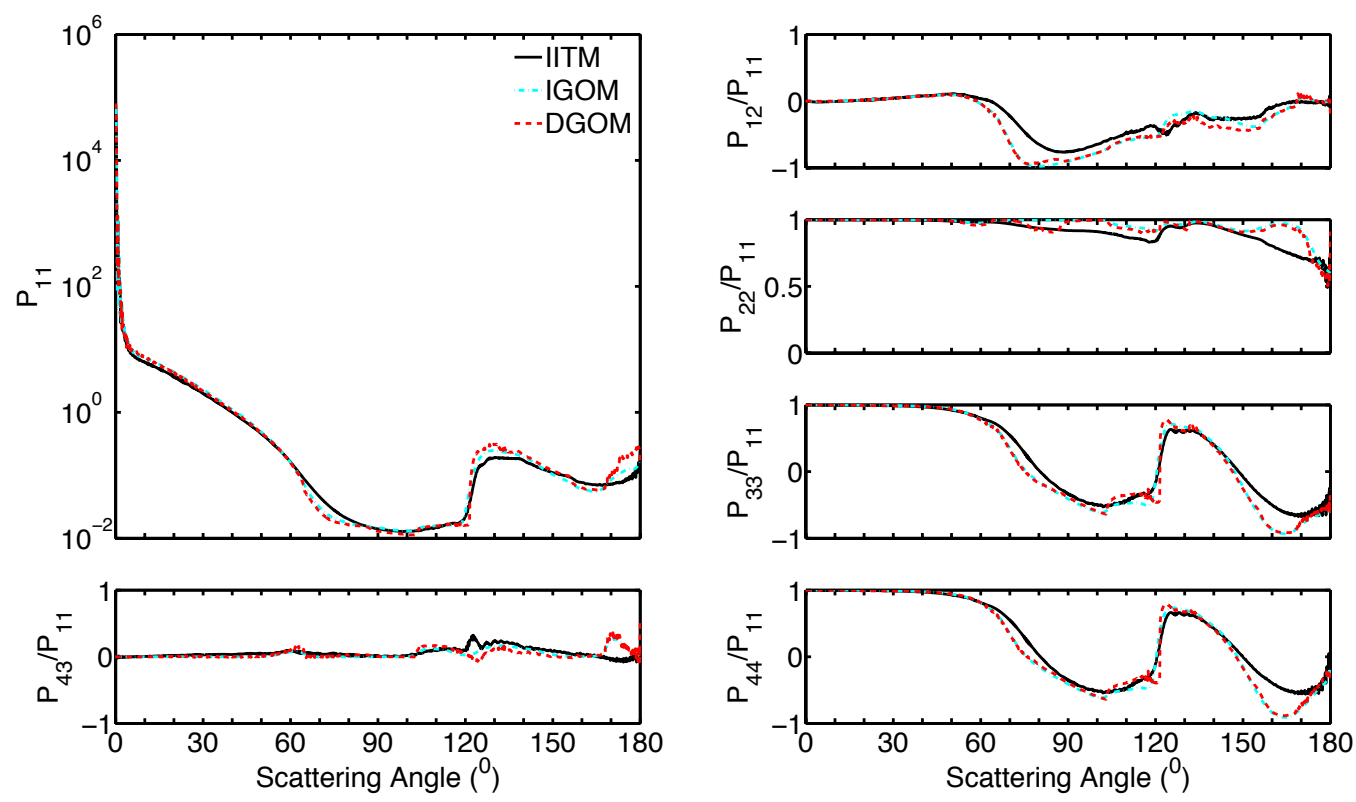

Figure 10 Comparisons of the scattering matrix elements of an oblate spheroid calculated in random orientation by IITM, IGOM, and DGOM. The incident wavelength is $0.628 \mu \mathrm{m}$. The lengths of its semi-major and semi-minor axes are $44.46 \mu \mathrm{m}$ and $39 \mu \mathrm{m}$. The refractive index of the spheroid is $1.33+\mathrm{i} 0.0$. 

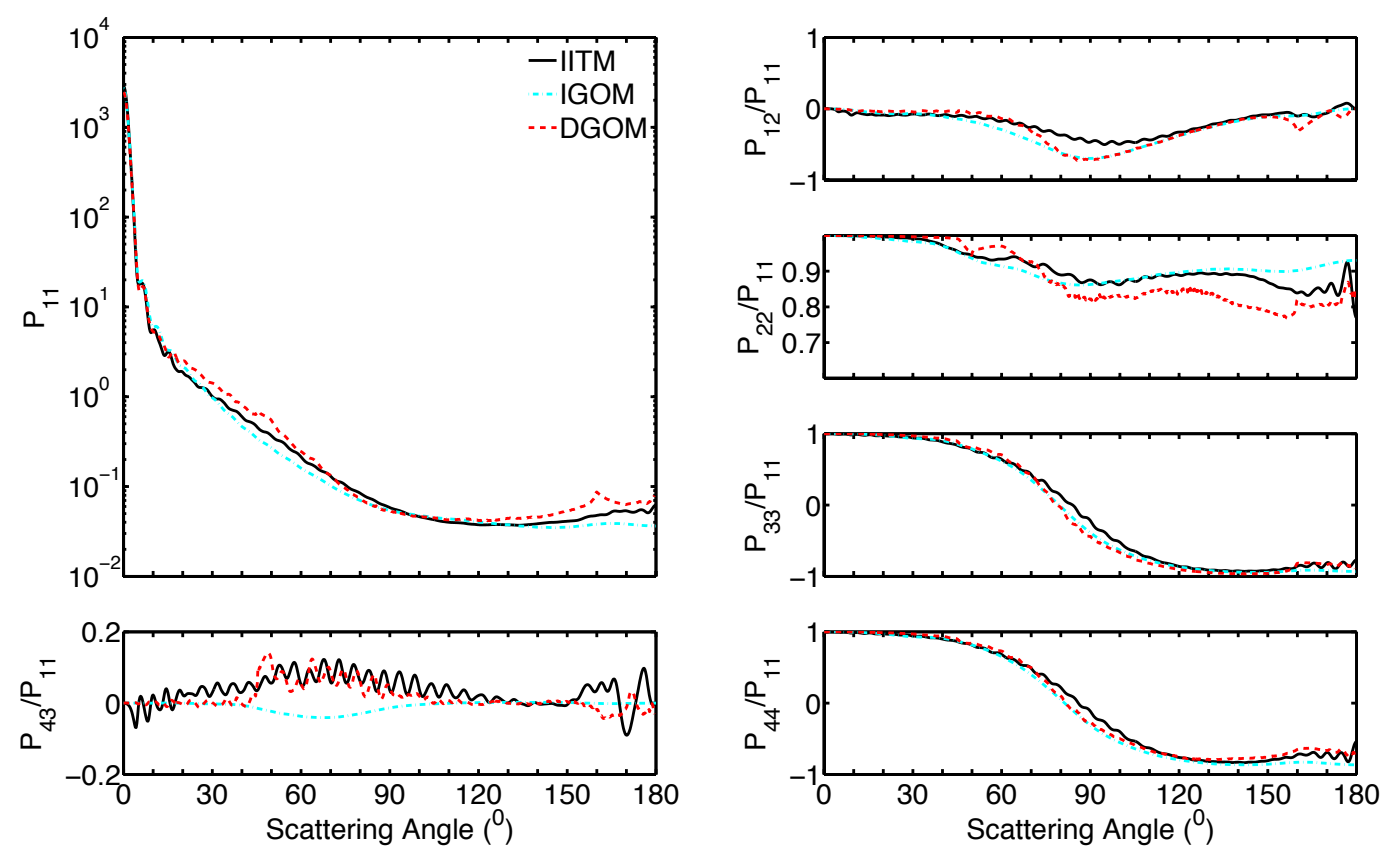

Figure 11 Comparisons of the scattering matrix elements of a prolate spheroid calculated in random orientation by IITM, IGOM, and DGOM. The incident wavelength is $0.628 \mu \mathrm{m}$. The lengths of its semi-major and semi-minor axes are $10 \mu \mathrm{m}$ and $4 \mu \mathrm{m}$. The refractive index of the spheroid is $1.53+\mathrm{i} 0.008$. 
References:

[1] van de Hulst HC. Light scattering by small particles: Dover publications, Inc.. New York, NY; 1981.

[2] Bohren CF, Huffman DR. Absorption and scattering of light by small particles: Wiley-Vch Verlag GmbH, Weinhim, Germany; 2008.

[3] Purcell EM, Pennypacker CR. Scattering and absorption of light by nonspherical dielectric grains. Astrophys. J. 1973;186:705-14.

[4] Draine BT. The discrete-dipole approximation and its application to interstellar graphite grains. Astrophys. J. 1988;333:848-72.

[5] Yurkin MA, Hoekstra AG. The discrete dipole approximation: an overview and recent developments. J. Quant. Spectrosc. Radiat. Transfer. 2007;106:558-89.

[6] Yee K. Numerical solution of initial boundary value problems involving Maxwell's equations in isotropic media. IEEE Trans. Antennas Propag. on. 1966;14:302-7.

[7] Taflove A, Hagness SC. Computational electrodynamics. Boston London: Artech house; 2000.

[8] Yang P, Liou K. Finite-difference time domain method for light scattering by small ice crystals in three-dimensional space. J. Opt. Soc. Am. A 1996;13:2072-85.

[9] Liu Q. The PSTD algorithm: A time - domain method requiring only two cells per wavelength. Microwave and Opt. Technology Lett. 1998;15:158-65.

[10] Liu QH. The pseudospectral time-domain (PSTD) algorithm for acoustic waves in absorptive media. Ultrasonics, IEEE Trans. Ferroelectrics and Frequency Control, 1998;45:1044-55. 
[11] Chen G, Yang P, Kattawar GW. Application of the pseudospectral time-domain method to the scattering of light by nonspherical particles. J. Opt. Soc. Am A 2008;25:785-90.

[12] Waterman P. Matrix formulation of electromagnetic scattering. Proceedings of the IEEE. 1965;53:805-12.

[13] Waterman P. Symmetry, unitarity, and geometry in electromagnetic scattering. Physical review D. 1971;3:825.

[14] Mishchenko MI, Travis LD, Lacis AA. Scattering, absorption, and emission of light by small particles: Cambridge university press, Cambridge, UK; 2002.

[15] Johnson BR. Invariant imbedding T matrix approach to electromagnetic scattering. Appl. opt. 1988;27:4861-73.

[16] Bi L, Yang P, Kattawar GW, Mishchenko MI. Efficient implementation of the invariant imbedding T-matrix method and the separation of variables method applied to large nonspherical inhomogeneous particles. J. Quant. Spectrosc. Radiat. Transfer. 2013;116:169-83.

[17] Macke A, Mueller J, Raschke E. Single scattering properties of atmospheric ice crystals. J. Atmos. Sci. 1996;53:2813-25.

[18] Yang P, Liou KN. Geometric-optics - integral-equation method for light scattering by nonspherical ice crystals. Appl. Opt. 1996;35:6568-84.

[19] Bi L, Yang P, Kattawar GW, Hu Y, Baum BA. Scattering and absorption of light by ice particles: Solution by a new physical-geometric optics hybrid method. J. Quant. Spectrosc. Radiat. Transfer. 2011;112:1492-508. 
[20] Hovenac EA, Lock JA. Assessing the contributions of surface waves and complex rays to far-field Mie scattering by use of the Debye series. J. Opt. Soc. Am A 1992;9:78195.

[21] Davis GE. Scattering of light by an air bubble in water. J. Opt. Soc. Am. $1955 ; 45: 572-81$.

[22] Sentis MPL, Onofri FRA, Méès L, Radev S. Scattering of light by large bubbles: Coupling of geometrical and physical optics approximations. J. Quant. Spectrosc. Radiat. Transfer. 2016;170:8-18.

[23] Lock JA. Ray scattering by an arbitrarily oriented spheroid. I. Diffraction and specular reflection. Appl. opt. 1996;35:500-14.

[24] Lock JA. Ray scattering by an arbitrarily oriented spheroid. II. Transmission and cross-polarization effects. Appl. opt. 1996;35:515-31.

[25] Ren KF, Onofri F, Rozé C, Girasole T. Vectorial complex ray model and application to two-dimensional scattering of plane wave by a spheroidal particle. Opt. lett. 2011;36:370-2.

[26] Deschamps GA. Ray techniques in electromagnetics. Proceedings of the IEEE. 1972;60:1022-35.

[27] Jiang K, Han Xe, Ren KF. Scattering of a Gaussian beam by an elliptical cylinder using the vectorial complex ray model. J. Opt. Soc. Am. A 2013;30:1548-56.

[28] Yang M, Wu Y, Sheng X, Ren KF. Comparison of scattering diagrams of large nonspherical particles calculated by VCRM and MLFMA. J. Quant. Spectrosc. Radiat. Transfer. 2015;162:143-53. 
[29] Onofri FRA, Ren KF, Sentis M, Gaubert Q, Pelcé C. Experimental validation of the vectorial complex ray model on the inter-caustics scattering of oblate droplets. Opt. express. 2015;23:15768-73.

[30] O'Neill B. Elementary differential geometry: Academic press, San Diego, California; 2006.

[31] Yang P, Liou K. Light scattering by hexagonal ice crystals: solutions by a ray-by-ray integration algorithm. J. Opt. Soc. Am. A 1997;14:2278-89.

[32] Bi L, Yang P, Kattawar GW, Kahn R. Single-scattering properties of triaxial ellipsoidal particles for a size parameter range from the Rayleigh to geometric-optics regimes. Appl. Opt. 2009;48:114-26.

[33] Tao T. Differential forms and integration. Tech. Rep., Department of Mathematics, UCLA; 2007.

[34] Yan W-Z, Du Y, Wu H, Liu D, Wu B-I. EM scattering from a long dielectric circular cylinder. Progress In Electromagnetics Research. 2008;85:39-67.

[35] Sun B, Yang P, Kattawar GW. Many-Body Iterative T-matrix Method for Large Aspect Ratio Particles. J. Quant. Spectrosc. Radiat. Transfer. 2013;127: 165-75. 